\title{
RECENT UPGRADES AND EXTENSIONS OF THE ASDEX UPGRADE ECRH SYSTEM
}

\author{
D. Wagner ${ }^{1}$, J. Stober ${ }^{1}$, F. Leuterer ${ }^{1}$, F. Monaco ${ }^{1}$, M. Münich ${ }^{1}$, \\ D. Schmid-Lorch ${ }^{1}$, H. Schütz ${ }^{1}$, H. Zohm ${ }^{1}$, M. Thumm ${ }^{2}$, \\ T. Scherer ${ }^{3}$, A. Meier ${ }^{3}$, G. Gantenbein ${ }^{2}$, J. Flamm², \\ W. Kasparek ${ }^{4}$, H. Höhnle ${ }^{4}$, C. Lechte ${ }^{4}$, A.G. Litvak ${ }^{5}$, \\ G.G. Denisov ${ }^{5}$, A. Chirkov ${ }^{5}$, L.G. Popov ${ }^{6}$, V.O. Nichiporenko ${ }^{6}$, \\ V.E. Myasnikov ${ }^{6}$, E.M. Tai ${ }^{6}$, E.A. Solyanova ${ }^{6}$, S.A. Malygin ${ }^{6}$ \\ ${ }^{1}$ Max-Planck-Institut für Plasmaphysik, EURATOM-IPP, Boltzmannstr.2, \\ D-85748 Garching, Germany \\ e-mail: dietmar.wagner@ipp.mpg.de \\ Karlsruhe Institute of Technology, EURATOM-KIT \\ ${ }^{2}$ Institut fürHochleistungsimpuls- und Mikrowellentechnik, \\ ${ }^{3}$ Institut für Materialforschung PO Box 3640, D-76021 Karlsruhe, Germany \\ ${ }^{4}$ Institut für Plasmaforschung, Universität Stuttgart, D-70569 Stuttgart, \\ Germany \\ ${ }^{5}$ Institute of Applied Physics, 46 Ulyanov St., Nizhny Novgorod, 603950, \\ Russia \\ ${ }^{6}$ GYCOM Ltd, 46 Ulyanov St., Nizhny Novgorod, 603155, Russia
}

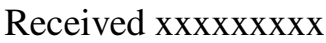

\begin{abstract}
The multi-frequency Electron Cyclotron Heating (ECRH) system at the ASDEX Upgrade tokamak employs depressed collector gyrotrons, step-tunable in the range 105-140 GHz. The system is equipped with a fast steerable launcher allowing for remote steering of the ECRH RF beam during the plasma discharge. The gyrotrons and the mirrors are fully integrated in the discharge control system. The polarization can be controlled in a feed-forward mode. 3 Sniffer probes for millimeter wave stray radiation detection have been installed.
\end{abstract}

Key words: Electron cyclotron resonance heating, step-tunable gyrotron, fast steerable launcher. 


\section{Introduction}

Electron cyclotron resonance heating (ECRH) and current drive (ECCD) experiments at the ASDEX Upgrade tokamak first started in 1996 with a single frequency $(140 \mathrm{GHz})$ system that comprises 4 gyrotrons with $0.5 \mathrm{MW} / 2 \mathrm{sec}$ each (optionally $0.7 \mathrm{MW} / 1 \mathrm{sec}$ ) and 4 independent transmission lines and launchers [1]. A new multi-frequency ECRH system is currently under construction that employs depressed collector gyrotrons, step-tunable in the range $105-140 \mathrm{GHz}$ with $1 \mathrm{MW}$ output power at $140 \mathrm{GHz}$ and slightly reduced power $(\sim 800 \mathrm{~kW})$ at lower frequencies [2]. The pulse length of the system is 10 $\mathrm{s}$, corresponding to the maximum flat top time of ASDEX Upgrade plasma discharges. In its final stage it will consist of 4 gyrotrons, where the first 3 gyrotrons are two-frequency gyrotrons, operating at 105 and $140 \mathrm{GHz}$. For the fourth gyrotron a broadband output window is under development that will allow operation also at intermediate frequencies. The transmission line consists of a quasi-optical Matching Optics Unit (MOU) and of non-evacuated corrugated $\mathrm{HE}_{11}$ waveguides with an inner diameter of $87 \mathrm{~mm}$ and a total length of approximately $70 \mathrm{~m}$. The launchers of the new system have a poloidal fast steering capability that allows for a change of the deposition location during the discharge without changing the toroidal magnetic field. The ultimate goal is to have a very flexible system for localized plasma heating and current drive that allows for feedback control of neoclassical tearing modes, pressure profile and transport [3].

Since 2007 ASDEX Upgrade operates with fully tungsten covered plasma facing components. Central heating with ECRH plays a key role in suppressing tungsten accumulation in the plasma center. Up to now the standard operation mode is the extraordinary mode at the second harmonic, X2-mode, because of its full single pass absorption. In order to extend the applicability of central ECRH to a wider range of magnetic field and plasma current, schemes with reduced single-pass absorption have been implemented: X3-mode heating allows to reduce the magnetic field by $30 \%$, such that the first $\mathrm{H}$-modes with an ITER-like safety factor of $\mathrm{q}_{95}=3$ could be run. Heating with the second harmonic ordinary mode, $\mathrm{O} 2$-mode, increases the plasma cutoff density for the ECRH by a factor of 2 and therefore allows to access higher plasma currents and triangularities [4].

\section{Frequency Step-Tunable Gyrotrons}

Three gyrotrons of the multi-frequency system are two-frequency GYCOM gyrotrons, working at $105 \mathrm{GHz}$ and at $140 \mathrm{GHz}$ with the $\mathrm{TE}_{17,6}$ and $\mathrm{TE}_{22,8}$ cavity modes respectively. They are equipped with a single-disk CVD (Chemical Vapor Deposition) diamond vacuum window which is resonant at 
both frequencies. The fourth gyrotron is supposed to be step-tunable, i.e. it can work at additional frequencies within the same frequency range. Because of the space requirement for additional phase correcting mirrors in the MOU only two additional frequencies are foreseen at $117 \mathrm{GHz}$ and $127 \mathrm{GHz}$. This gyrotron requires a broadband output window. Several options for this vacuum window are under investigation, including a Brewster window [5,6] and CVD diamond discs with matched corrugated surfaces [7].

\section{Broadband Transmission Line}

The MOU connected to each gyrotron (Fig.1) contains a pair of phase correcting mirrors for each frequency. They are mounted on turntables and automatically switched into the beam path for each frequency. These mirrors also provide the alignment of the gyrotron output beams of the individual frequencies onto the optical axis of the transmission line which remains the same for all frequencies. One set of grooved polarizer mirrors with groove depths of $\lambda / 4$ and $\lambda / 8$ ( $\lambda$ is the vacuum wavelength) scaled to the center frequency of $122.5 \mathrm{GHz}$ allows to provide any arbitrary polarization over the full frequency band of the system $(105-140 \mathrm{GHz})$ [8]. Power monitoring is done by a directional coupler in the second phase correcting mirror. Each MOU also contains a short-pulse calorimetric load which can be connected to the gyrotron by two switching mirrors. There is also one long-pulse load installed for all 4 gyrotrons of the new system to which each of the 4 gyrotrons can be connected. A focusing mirror couples the beam into a corrugated $\mathrm{HE}_{11}$ waveguide transmission line with I.D. $=87 \mathrm{~mm}$ with a total length of about 70 $\mathrm{m}$. The transmission is in normal air. After some conditioning, arcing in the waveguide was no problem at both frequencies for pulses with full power and pulse length. A second calorimetric load is installed at the torus, next to the torus window. The transmission efficiency of the system is measured by comparing the measured absorbed power in both loads. The torus load is also used to test the performance of the transmission line on each experimental day before plasma experiments start. The last miter bend mirror next to the torus window also contains a directional coupler. This signal is used for calibration of the polarizers. The geometrical arrangement of the miter bend directional coupler is such that its output corresponds to the vertical component of the electrical field of the waveguide mode, which varies as a function of the polarization. It serves also as a power monitor during the gyrotron pulse. The torus vacuum windows for the transmission lines with two-frequency gyrotrons are single disc CVD diamond discs. For the four-frequency gyrotron a remotely tunable double disc window [9] has been developed and installed at ASDEX Upgrade. A Brewster type window can not be used at this position, since it has to be transparent independent of the polarization of the transmitted beam. 


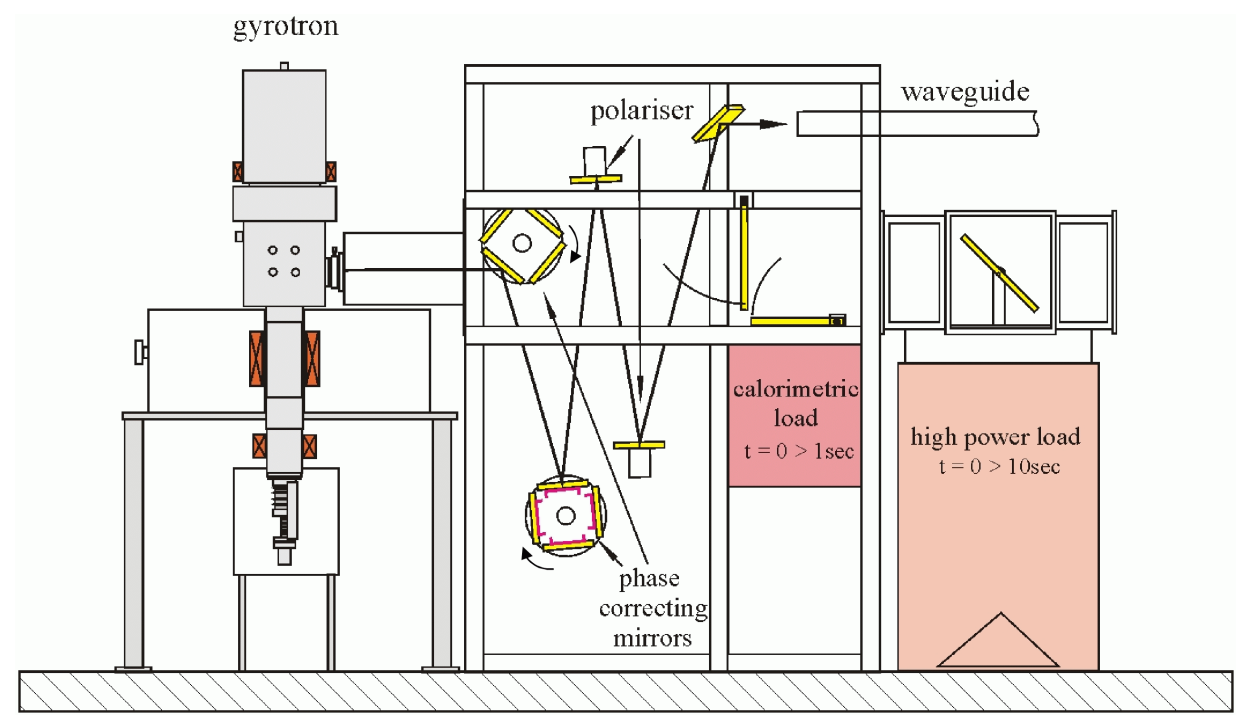

Fig.1 Schematic of gyrotron with MOU, 4-frequency version.

\section{Recent System Extensions}

\subsection{New remote control features}

In the past all parameters of the ECRH system including the timing of the gyrotron and the angles of the launcher and the polarizer mirror angles had to be set before the plasma pulse. The new system allows the triggering of the ECRH system by the main discharge control system (DCS) of ASDEX Upgrade depending on plasma conditions. An example is given in Fig.2. Here the ECRH is switched on by the DCS. The main part of the time delay corresponds to the cycle time of the DCS $(1.85 \mathrm{~ms})$. The time for the cathode voltage ramp-up is $500 \mu \mathrm{s}$. The body voltage is switched on with a $200 \mu \mathrm{s}$ delay in order to limit the voltage depression in the gyrotron which would otherwise lead to excessive body current during the voltage ramp-up. An important application is the feedback control of impurities in the plasma center, e.g. central heating by ECRH can be automatically added in the discharge if the tungsten concentration in the plasma center exceeds certain limits that would lead to excessive radiation losses. The modulation frequency is limited by the DCS cycle time to $500 \mathrm{~Hz}$. Higher modulation frequencies up to $25 \mathrm{kHz}$ are possible by directly triggering the gyrotron pulse with a diagnostic signal, e.g. the measured current in the Mirnov coils. At such high modulation frequencies it is impossible to run the depressed collector gyrotrons in a switch on and off mode. In this case the beam voltage is only reduced to a certain value where the output power is reduced down to $10 \%$, while the body voltage stays constant 
and the gyrotron mode is still oscillating. This is being applied for the stabilization of neoclassical tearing modes (NTM), where the gyrotron is modulated synchronously with the rotating magnetic island in the plasma, allowing for current drive only in the O-point of the magnetic island [10]. At this point launcher and polarizer mirror angles can only be controlled in a feed forward mode (Fig.3). The response times for the mirror drives are $200 \mathrm{~ms}$ for the polarizer mirrors and $18 \mathrm{~ms}$ for the launcher mirror. In a next step the poloidal launcher angle will be included in a feedback control loop for NTM stabilization.

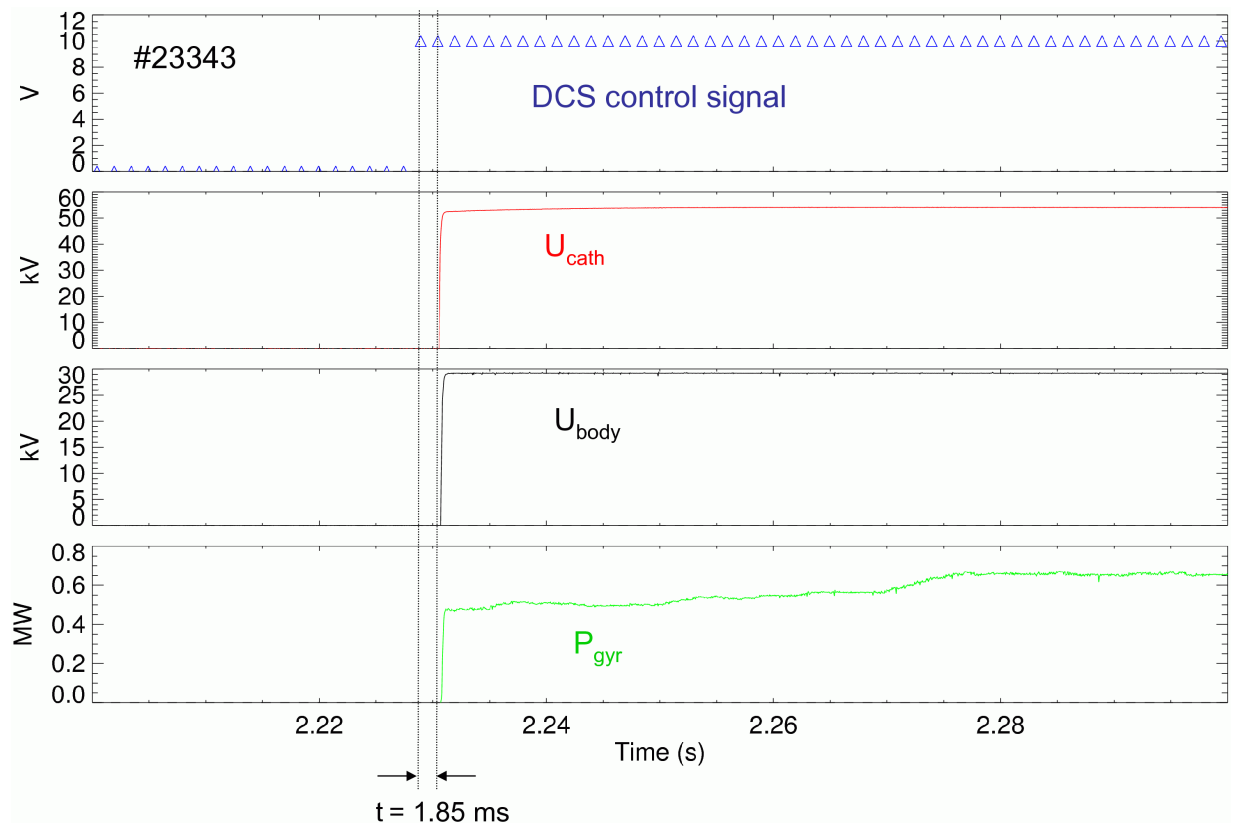

Fig.2 Plasma discharge with ECRH power controlled by the discharge control system (DCS).

\subsection{Holographic reflectors at the inner wall}

Up to now ECRH at ASDEX Upgrade was applied in the X2-mode. This heating scenario is limited by the cutoff electron density $\left(1.22 \cdot 10^{20} / \mathrm{m}^{3}\right.$ for $\mathrm{B}_{\mathrm{t}}=$ $2.5 \mathrm{~T}, \mathrm{f}=140 \mathrm{GHz}, \mathrm{T}_{\mathrm{e}}=2 \mathrm{keV}$ ). To access plasmas with higher densities, heating with the O2-mode is an option [4]. In a proof of principle experiment, $\mathrm{X} 2$-heating was used to pre-heat the plasma at densities below the X2-cutoff. When the density increased, the polarization was changed for $\mathrm{O} 2$-mode heating during the discharge and the pulse continued (Fig.3). The measurement of the 
central electron temperature in Fig. 3 was limited by an ECE cutoff ( $f=130.7$ $\mathrm{GHz}$ ) of the center most channel at a plasma radius $\rho=0.2$ between $\mathrm{t}=2.5 \mathrm{~s}$ and $3.2 \mathrm{~s}$. The same happened after $4 \mathrm{~s}$. Still it can be seen from this first experiment that the electron temperature stays almost constant when the polarization is changed to $\mathrm{O} 2$-mode. Unlike the $\mathrm{X} 2$-mode which is fully absorbed at the resonant surface in the plasma, heating in the $\mathrm{O} 2$-mode exhibits only partial absorption of approximately $70 \%$ of the injected power. After this first proof of principle, two tiles of the inner wall have been replaced with holographic reflectors that reflect the non-absorbed part of the beam again with O2-mode polarization allowing for a second pass through the resonant layer (Fig.4). That way the absorption can be increased up to $90 \%$. The holographic reflector is equipped with thermocouples underneath its surface, close to the edges. First successful measurements of the beam position using feed forward controlled launcher movements were performed. In future these thermocouples will give a feedback signal to the fast steerable launcher in order to make sure that the beam stays in the center of the reflector, even when the plasma density changes.

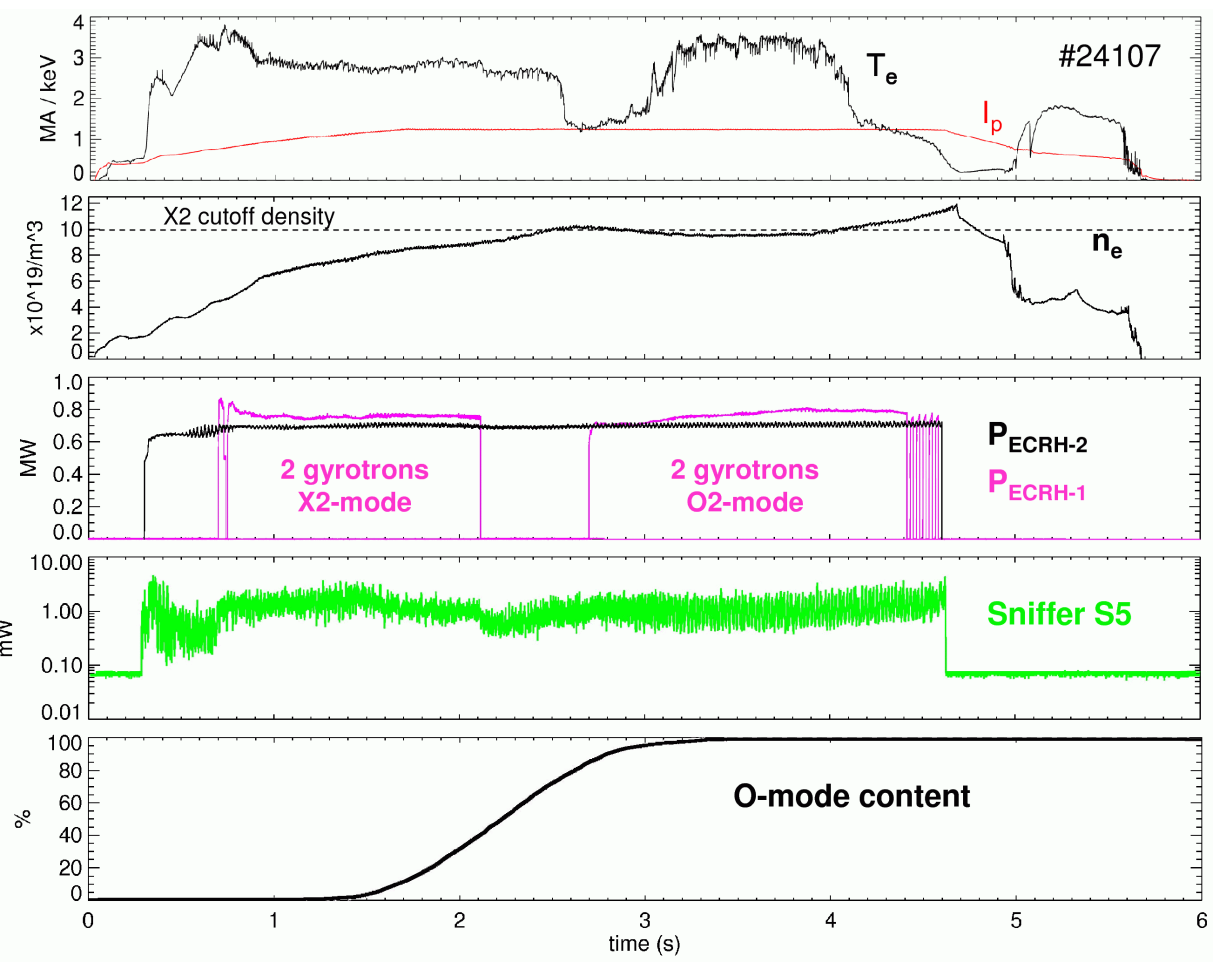

Fig.3 Parameters of ASDEX Upgrade discharge \#24107. Plasma current $I_{p}$, central electron temperature $T_{e}$, central electron density $n_{e}$, injected ECRH 
power of the old $\left(\mathrm{P}_{\mathrm{ECRH}-1}\right)$ and the new ECRH system $\left(\mathrm{P}_{\mathrm{ECRH}-2}\right)$, the signal from the sniffer probe in sector 5 and the calculated O2-mode content. The frequency of both ECRH systems was $140 \mathrm{GHz}$. The first two beams of the old system (ECRH-1) were polarized for X2-mode and the two later beams were polarized for O2-mode. The polarization of ECRH-2 started with X2-mode and was changed from $\mathrm{X} 2$ to $\mathrm{O} 2$ between $\mathrm{t}=1.1 \mathrm{~s}$ and $\mathrm{t}=2.7 \mathrm{~s}$.

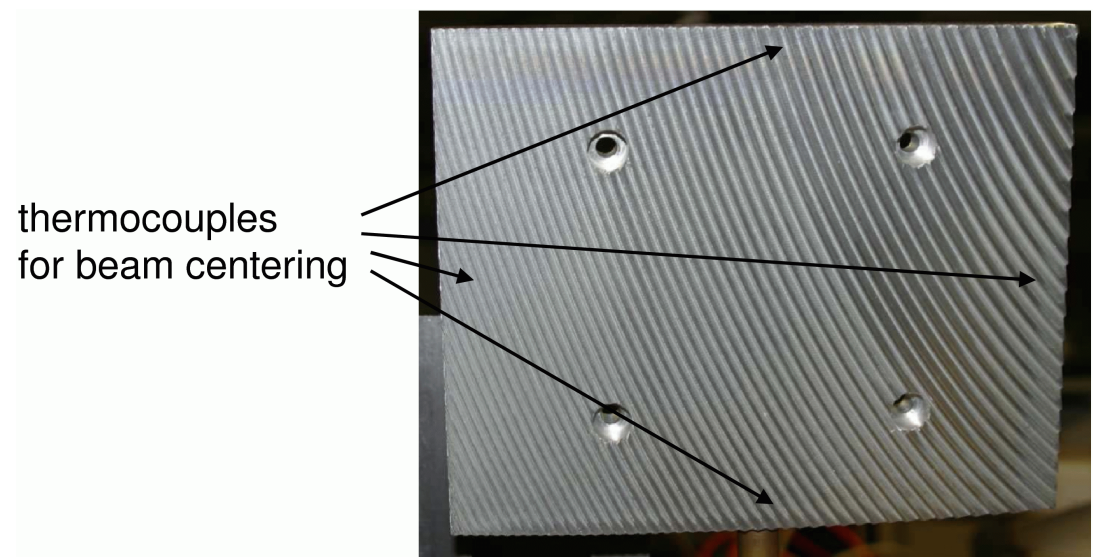

Fig.4 ASDEX Upgrade inner wall tile with holographic reflector grating and indicated positions of the thermocouples.

\subsection{Installation of sniffer probes for ECRH stray radiation detection}

Even prior to dedicated $\mathrm{O} 2$ and $\mathrm{X} 3$ heating experiments some hazards occurred due to ECRH operation caused by unexpected plasma conditions such as cutoffs or operational errors, resulting in broken pick up coils, burned insulators and molten steel. An example for an operational error is the injection of the ECRH beam with the wrong handedness of its elliptical polarization. The mistake was only discovered when glowing in a gap between two tiles of the inner wall was observed by one of the cameras whenever the ECRH was switched on. The beam was supposed to be launched in X2-mode polarization, but there was obviously a substantial part of non-absorbed power. To check the handedness of the polarization one of the polarizer mirrors was then rotated during a discharge. Fig 5 summarizes the result. The blue curve shows the calculated $\mathrm{O} 2$-mode content of the beam (which is only partially absorbed) and the foreseen X2-mode operating point for the originally calculated polarization, while the red curve shows the $\mathrm{O} 2$-mode content for the a polarization with the same ellipticity but inverted handedness. The green trace shows a $140 \mathrm{GHz}$ detector signal picked up via one of the ECE channels. The detected ECE signal was only slightly above the noise level and way below the interlock 
treshhold of the ECE system. Also shown is a sequence of photos showing the presence or absence of glowing events at the inner wall of the ASDEX Upgrade vessel during this polarization scan. The correlation shows that the original handedness was wrong. Since the pulse was not stopped by the ECE interlock system, an independent stray radiation detection system became a high priority issue. In a first step, one sniffer probe for millimetre wave stray radiation $[11,12]$ was installed in sector 5 next to the ECRH-2 launchers. Contrary to the detection via an ECE channel, it has a broad and polarization insensitive radiation characteristic. Although it proved to be useful as an interlock at several events, e.g. ECRH cutoffs with perpendicular injection, Fig.3 demonstrates that there is also a directivity of the stray radiation. In spite of the low absorption of only about $70 \%$ in the second part of the pulse, the sniffer probe delivered an almost constant signal and the ECRH was not switched off. The reason for this was the toroidal injection angle of $-15^{\circ}$. In this case the stray radiation has to travel all around the torus before it arrives back at the sniffer probe with multiple reflections at the torus wall as well as multiple passes through the plasma with partial absorption in between. Two additional sniffer probes were obtained from CNR Milano and installed approximately $90^{\circ}$ apart around the torus (Fig.6) [13]. The goal is to define thresholds for excessive stray radiation for various injection angles. Fig.7 shows the measured sniffer probe signals for a plasma discharge in which the O2-mode content was varied by rotating one of the polarizer mirrors. Further use for the optimization of the polarization adjustment is being investigated as well [13]. 


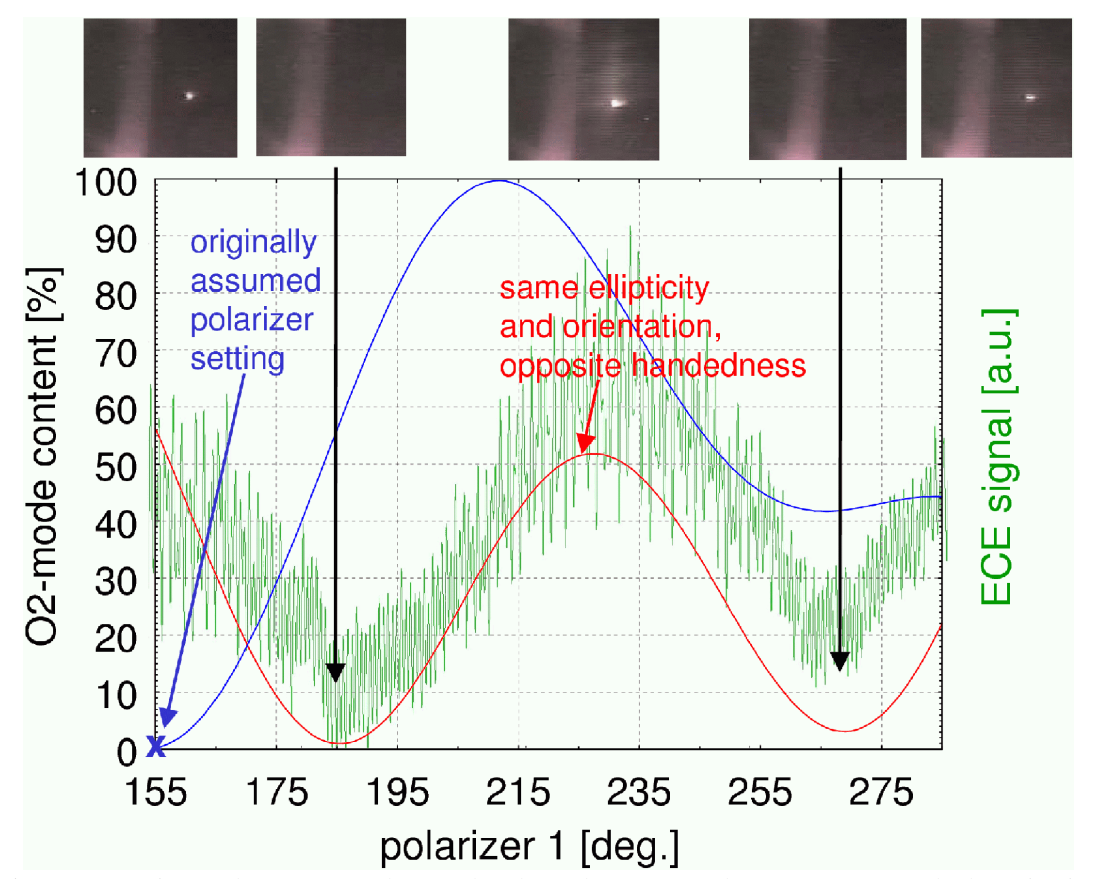

Fig.5 Comparison between the calculated O2-mode content and the timing of light emission at the inner wall for the originally assumed and the corrected handedness of the polarization of the ECRH beam. Also shown is a stray radiation signal collected via one of the ECE channels.

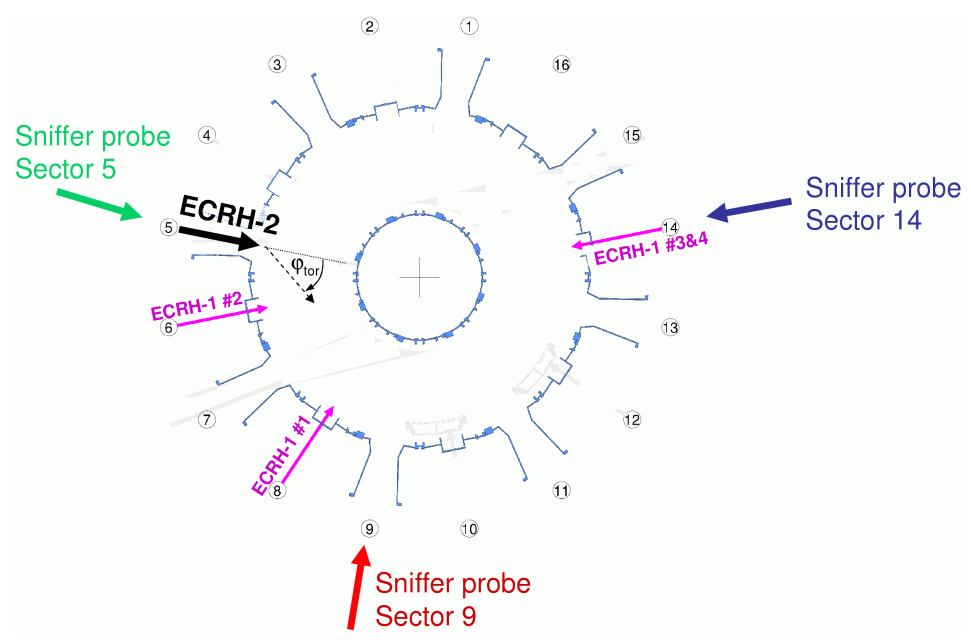

Fig.6 Top view of ASDEX Upgrade torus with indicated positions of the ECRH antennas and of the sniffer probes for stray radiation detection. 


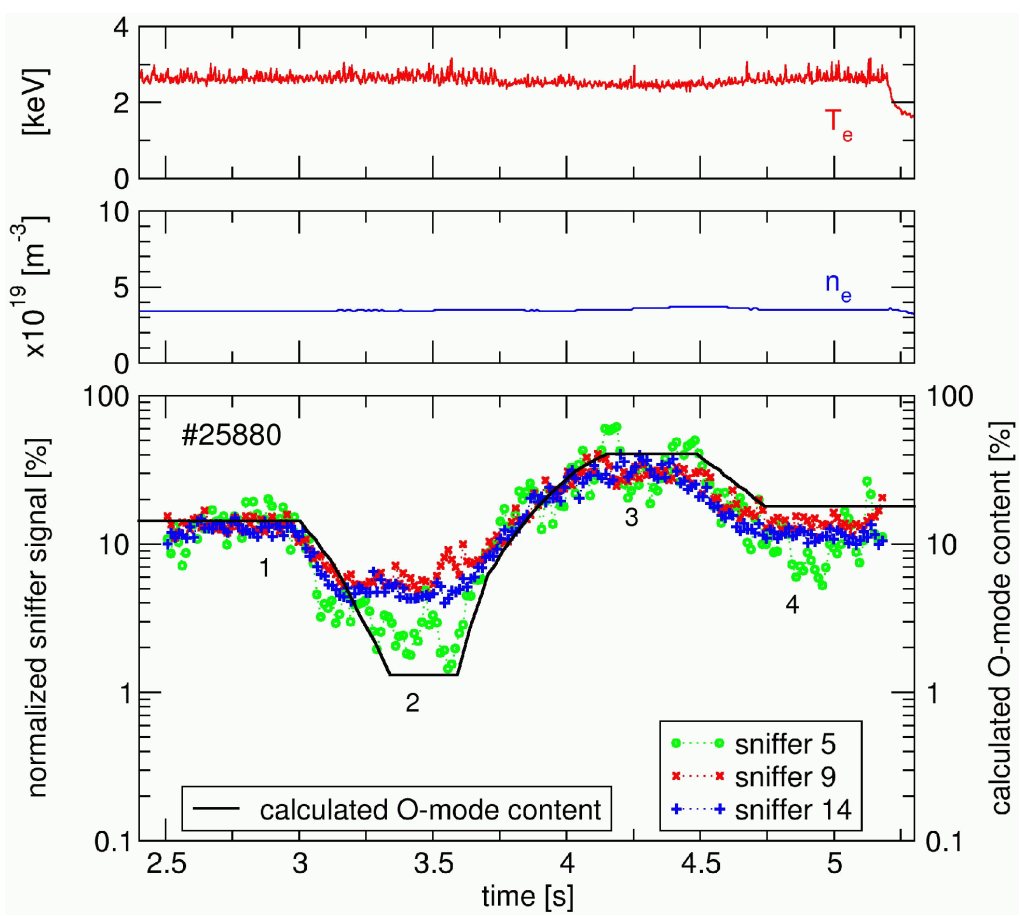

Fig.7 Central electron temperature $T_{e}$, central electron density $n_{e}$, calculated O2-mode coupling and measured signals from the 3 sniffer probes for $\mathrm{B}_{\mathrm{t}}=+2.5 \mathrm{~T}, \mathrm{I}_{\mathrm{p}}=-1 \mathrm{MA}$, launcher angles $\vartheta_{\mathrm{pol}}=-17^{\circ}$ and $\varphi_{\mathrm{tor}}=0^{\circ}$ with polarization modification during the discharge.

\section{Future Plans}

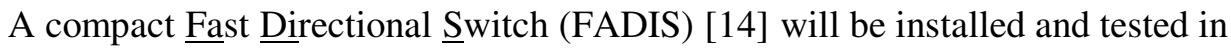
the ASDEX Upgrade ECRH transmission line. It is a compact shielded system, compatible with the $\mathrm{HE}_{11}$-waveguide transmission line (Fig.8). It can be used either as a beam combiner or as a beam switch between two transmission lines. The latter is especially interesting for the stabilization of neoclassical tearing modes, because such electronically controlled switching of one beam between two antennas, synchronous to the rotation of the magnetic islands in the tokamak plasma, would maximize the efficiency for stabilization of these modes. Another foreseen application using two gyrotrons connected to the FADIS is to control two experiments at the same time. E.g. to use one beam for NTM stabilization and the other one for central heating. Successful high power tests with a prototype of FADIS have been performed at IPP Greifswald [14]. 


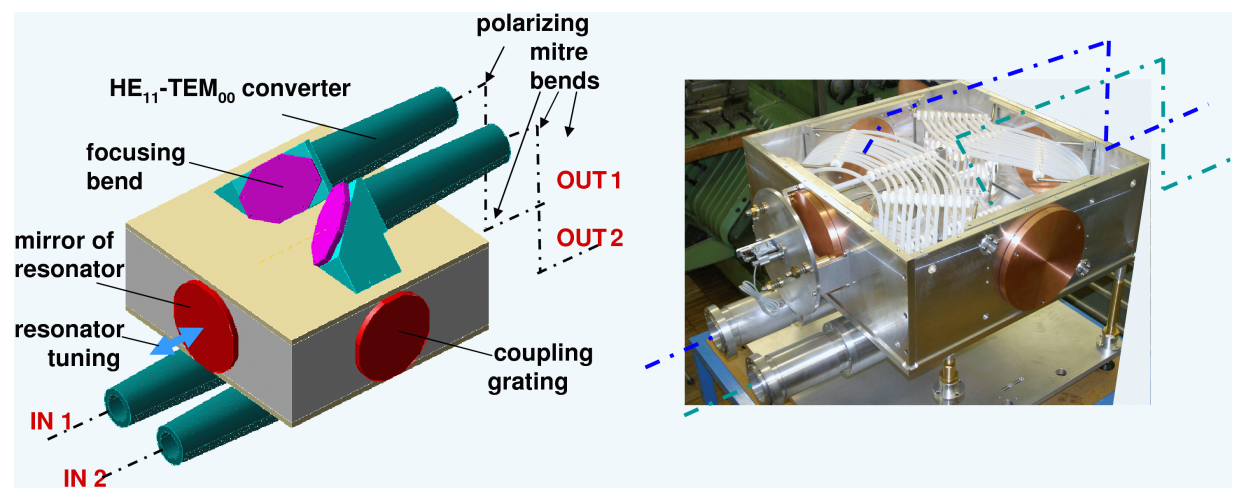

Fig.8 Sketch of the compact fast directional switch (FADIS) (left) and picture of the prototype (right).

\section{References}

[1] F. Leuterer, et al., "Operation experience with the ASDEX Upgrade ECRH system”, Fusion Science and Technology 55 (2009), 31-44.

[2] D. Wagner et al., "Present Status of the New Multifrequency ECRH System for ASDEX Upgrade"IEEE Trans. Plasma Science 36 (2008), 324331.

[3] A. Manini et al., "Development of a feedback system to control MHD instabilities in ASDEX Upgrade", Fus. Eng. Design, 82 (2007), 995-1001.

[4] H. Höhnle et al., "Investigation of the O2- and X3-mode heating in ASDEX Upgrade", 36th EPS Conf. on Plasma Phys. 33E (2009),1.145.

[5] G.G.Denisov et al., "Multi-Frequency Gyrotron with BN Brewster Window", Conf. Digest of the Joint 31th International Conference on Infrared and Millimeter Waves and 14th International Conference on Terahertz Electronics, Shanghai, China (2006), 75.

[6] O. Prinz et al., "Matching the output beam of a multi-frequency gyrotron to a Brewster window with small aperture", 8th IEEE International Vacuum Electronics Conference (IVEC 2007), pp. 49-50, Kitakyushu, Japan, 2007.

[7] V.I. Belousov et al., "Broad band matched windows for gyrotrons", Conference Digest, Joint $34^{\text {th }}$ Int. Conf. on Infrared and Millimeter and Terahertz Waves, Busan, Korea (2009).

[8] D. Wagner and F. Leuterer, " Broadband Polarizers for High-Power MultiFrequency ECRH Systems", Int. J. on Infrared and Millimeter Waves 26 (2005), 163-172.

[9] R. Heidinger et al., "Tunable double disk window for ECH\&CD system of ASDEX Upgrade", Conference Digest, Joint $29^{\text {th }}$ Int. Conf. on Infrarared and Millimeter Waves and $12^{\text {th }}$ Int. Conf. on Terahertz Electronics, Karlsruhe, Germany (2004),723. 
[10] H. Zohm et al., "Control of MHD instabilities by ECCD: ASDEX Upgrade results and implications for ITER", Nuclear Fusion 47 (2007), 228-232

[11] S. Nowak et al., "Measurements of residual power of EC waves propagating in FTU tokamak ECRH experiments at $140 \mathrm{GHz}$ ", Fusion Eng. Design 53, 315-320, (2001).

[12] F. Gandini et al., "The detection of non-absorbed millimeterwave power during EC-heating and current drive", Fus. Eng. Design 56-57 (2001), 975-979.

[13] D. Wagner et al., "Feed forward polarization control during ECRH discharges at ASDEX Upgrade", Fusion Science and Technology, to be published.

[14] W. Kasparek et al., "A fast switch, combiner and narrow-band filter for high-power millimeter wave beams", Nuclear Fusion 48 (2008), 054010 . 